\title{
Demanda de trabajo del hogar remunerado en el Perú urbano
}

\author{
Cecilia Garavito*
}

\begin{abstract}
RESUMEN
El objetivo de este artículo es tanto analizar los determinantes de la probabilidad de contratar trabajo del hogar remunerado, como estimar la función de demanda de estos servicios por parte de los hogares. Dado que la mayoría de los trabajadores del hogar se encuentra en el sector urbano, vamos a analizar solamente la demanda de trabajo del hogar remunerado en dicho sector. Para esto emplearemos la Encuesta Nacional de Uso del Tiempo (ENNUT) y la Encuesta Nacional de Hogares (ENAHO), para el año 2010, y la ENAHO del año 2016. En cuanto a la probabilidad de contratar trabajo del hogar remunerado, encontramos que si bien la contratación de trabajo del hogar remunerado "cama adentro» se está reduciendo en relación al THR "cama afuera», este persiste en los hogares "mayores» y con más ingresos, mientras los hogares más jóvenes contratan en su mayoría trabajadoras del hogar por horas. Asimismo, mayores ingresos laborales y no laborales llevan a una mayor probabilidad de contratar THR en relación a no hacerlo, y los efectos marginales del ingreso laboral del cónyuge son mayores para la modalidad "cama adentro", sucediendo lo contrario para la modalidad "cama afuera». En cuanto a la demanda de trabajo del hogar remunerado, encontramos que las horas demandadas dependen negativamente de la tasa de salarios pagada a la trabajadora del hogar y positivamente de los ingresos familiares. Asimismo, existe un efecto sustitución entre el trabajo doméstico de los miembros y el de trabajadora del hogar remunerada. Adicionalmente, la presencia de mujeres en el hogar reduce las horas demandadas de THR «cama adentro». Finalmente, las jornadas laborales son mayores si el empleador vive en Lima Metropolitana, lo cual está relacionado a que este es el mayor mercado de trabajo del hogar remunerado en el Perú, donde también se encuentra la mayor oferta.
\end{abstract}

Palabras clave: mercado de trabajo, trabajo del hogar remunerado, demanda de trabajo, género. Clasificación JEL: J15, J16, J23, J46

\section{Demand for paid domestic labor in urban Peru}

\begin{abstract}
The aim of this article is both to analyze the determinants of the probability of demanding paid domestic work (PDW), and to estimate the demand of the households for these services. Given that that the majority of the domestic workers are in the cities, we are going to analyze this demand in the urbane area. We will work with the Encuesta Nacional de Uso del Tiempo
\end{abstract}

\footnotetext{
* Profesora principal del Departamento de Economía de la Pontificia Universidad Católica del Perú.
} 
(ENNUT) and the Encuesta Nacional de Hogares (ENAHO), for the years 2010, and with the ENAHO 2016. Regarding the probability of hiring domestic paid work, we find that even if the demand for live-in domestic work is falling if compared with live-out domestic work, that type of arrangement subsists for «older» and richer households, while the younger household hire mostly live-out paid domestic workers. We also find that higher labor and non-labor earnings have a positive effect on the probability of hiring PDW in relation to not hiring it, and the marginal effects of the labor earnings of the spouse are higher for live-in workers, and the effect is the opposite for live - out workers. Regarding the demand of paid domestic work, we find that the hours demanded have a negative relation with the wage paid to the domestic worker and a positive relation with the family earnings. More so, there is a substitution effect between the domestic work done by the members of the household and the hours worked by the paid domestic workers. Additionally, the presence of women in the household reduces the hours of live-in paid housework demanded. Finally, the labor journey is longer if the employer lives in Lima Metropolitana, which is related to the fact that the capital city is the greatest market for paid housework in Peru, and also where the majority of these workers are.

Keywords: Labor market, paid housework, labor demand, gender.

JEL Classification: J15, J16, J23, J46

\section{INTRODUCCIÓN}

La demanda por trabajo del hogar remunerado está estrechamente ligada a la economía del hogar. La organización de las tareas reproductivas dentro de un hogar muchas veces requiere mano de obra externa, debido a que los miembros de la familia no pueden o no están dispuestos a emplear parte de su tiempo en dichas tareas. De acuerdo a la Organización Internacional del Trabajo (OIT) (2013), el trabajo del hogar remunerado (THR) es una de las modalidades de lo que se llama trabajo doméstico remunerado en general; este incluye además los servicios de cuidado especializado de niños, adultos enfermos y mayores, y los servicios específicos para el hogar, tales como el manejo de autos y cuidado de jardines, entre otros. Lo que la OIT llama trabajo del hogar remunerado consiste entonces en las tareas diarias de limpieza, preparación de comidas y lavado y planchado de ropa, y constituye el rubro más importante del trabajo doméstico remunerado, oscilando alrededor del $90 \%$ del total.

En los países desarrollados y en las denominadas economías emergentes la demanda de trabajo del hogar remunerado está aumentando en los últimos años. Así, por ejemplo, Cox (2000) para el caso de Inglaterra, Stancanelli y Straton (2010) para Inglaterra y Francia, Lan (2003) para el caso de Taiwán, Cortes y Pan (2013) y Suen (1994) para Hong Kong, señalan que la migración de trabajadoras desde países menos desarrollados ha abaratado el costo de sus servicios, lo cual ha influido positivamente en la participación de las mujeres empleadoras en la fuerza laboral. En América Latina, la historia es diferente. En primer lugar, el porcentaje de trabajadoras del hogar remuneradas 
con respecto a la fuerza laboral ocupada se está reduciendo, y más aún en el caso de aquellas que ofrecen su trabajo bajo la modalidad «cama adentro" (ver Blofield, 2009; COMMCA, 2010; Garavito, 2015, 2017). En segundo lugar, las trabajadoras del hogar ya no son en su mayoría migrantes pobres de las zonas rurales que buscan salir de la pobreza ofreciendo sus servicios en las ciudades. El trabajo del hogar remunerado en las ciudades de América Latina es ahora una actividad llevada a cabo por mujeres pobres y poco calificadas que proceden de las zonas urbanas marginales. Finalmente, la mayor parte de estas trabajadoras ofrece sus servicios bajo la modalidad «cama afuera», es decir, no viven en el hogar de sus empleadores, esto contrasta con algunos programas de migración de trabajadoras en otras zonas del mundo que requieren que la trabajadora viva en el hogar de sus empleadores ${ }^{1}$.

En el caso del Perú, el trabajo del hogar remunerado constituye un porcentaje reducido de la fuerza laboral y se aprecia una ligera caída en dicho porcentaje en los últimos años. Es así que a nivel nacional, su participación en la población económicamente activa (PEA) ocupada ha caído de 3,1\% en 2010 a 2,4\% en 2016. En el sector urbano, donde se encuentran la mayoría de trabajadoras del hogar remuneradas, la caída ha sido bastante mayor: 4,4\% en 2010 a 2,8\% en 2016. Aun así es importante su estudio debido a que se trata de uno de los sectores laborales más vulnerables en nuestro país, con ingresos por debajo del salario mínimo, largas jornadas laborales, alto porcentaje de trabajo infantil — sobre todo en el área rural — y ausencia de contratos de trabajo y seguridad social provista por el empleador ${ }^{2}$.

En este trabajo vamos a analizar tanto la probabilidad de contratar trabajo del hogar remunerado, como los determinantes de las horas demandadas por los hogares. Para la primera estimación emplearemos los datos de la Encuesta Nacional de Uso del Tiempo 2010 (ENUT) y los datos de la Encuesta Nacional de Hogares (ENAHO) para los años 2010 y 2016. Para la segunda estimación emplearemos los datos de las ENAHO 2010 y 2016, ya que solamente estas encuestas nos permiten tener los datos de horas de trabajo y de ingresos de cada trabajadora del hogar.

Sostenemos que los recientes cambios en el mercado de trabajo del hogar remunerado estarían relacionados no solamente al mayor desarrollo del mercado laboral que ofrece otras alternativas de empleo a mujeres pobres y con bajo nivel de instrucción, sino también con razones de demanda tales como cambios en los ingresos del jefe del hogar y del cónyuge, asociados a los cambios en los patrones de participación por sexo en la fuerza laboral.

El objetivo de este artículo es entonces analizar la probabilidad de contratar trabajo del hogar remunerado, así como estimar la función de demanda de estos servicios

\footnotetext{
1 Elegimos emplear el género femenino ya que más del 95\% de los trabajadores del hogar remunerados son mujeres, sobre todo en el sector urbano que es el que vamos a estudiar.

2 Para un análisis de la oferta de trabajo del hogar remunerado ver Garavito, 2017.
} 
por parte de los hogares, tomando en cuenta no solamente los determinantes usuales de la demanda de servicios laborales tales como su costo y los ingresos de quienes lo demandan, sino también la participación laboral de los miembros del hogar. Dado que la mayoría de los trabajadores del hogar se encuentra en el sector urbano ${ }^{3}$ y que las tareas llevadas a cabo por estos trabajadores son distintas en ambos sectores ${ }^{4}$, vamos a analizar solamente la demanda de trabajo del hogar remunerado en el sector urbano.

En la segunda sección de este trabajo revisamos la literatura teórica y empírica sobre el tema. En la tercera sección presentamos el marco conceptual que nos permitirá analizar las relaciones entre la demanda de trabajo del hogar remunerado y las características de la familia demandante. En la cuarta sección presentamos estadísticas descriptivas sobre la demanda de trabajo del hogar remunerado, así como los estimados econométricos. Finalmente, en la quinta sección presentamos las conclusiones de este trabajo.

\section{ESTADO DE LA CUESTIÓN}

En esta sección revisamos en primer lugar el concepto de reproducción social, que enmarca el estudio de la demanda por trabajo del hogar remunerado. En segundo lugar revisamos la literatura sobre la economía del hogar y la demanda de trabajo del hogar remunerado. En tercer lugar presentamos el marco conceptual que nos permitirá estimar la probabilidad de contratar trabajo del hogar remunerado (THR), así como las horas de THR demandadas por los hogares.

Un primer punto a analizar es el concepto de reproducción social, el cual incluye las tareas de mantenimiento del hogar, entre otras. Siguiendo a Mattingly (2012), la reproducción social se define como la «reproducción sistemática de determinadas estructuras ideológicas, y el trabajo de sostener a los seres humanos a lo largo de su ciclo de vida ${ }^{5} . .$. . Es decir, la reproducción social depende no solamente de las necesidades diarias de un hogar, sino también de la estructura socioeconómica en la que este está inserto. En relación al trabajo necesario para las tareas de reproducción social, Duffy (2007) cita las definiciones de trabajo reproductivo de Laslett y Brener (1989): «a set of tasks necessary to maintain existing life and to reproduce the next generation» y de Glenn (1992): "work that maintains daily life and work that reproduces the next generation». A partir de ambas definiciones, Duffy separa el trabajo reproductivo entre aquel llevado a cabo en el hogar por personas que no pertenecen a este; y las tareas de reproducción social llevadas a cabo por organizaciones externas al hogar, tales como la enseńanza, el cuidado

3 El 84,1\% de las trabajadoras de hogar se encontraba en el área urbana en el año 2010. Ver Garavito, 2017. Ver también Instituto Nacional de Estadística e Informática, 2011.

4 El trabajo del hogar en el sector rural puede mezclarse con la cooperación de personas que no viven en el hogar en el trabajo productivo. Sobre los problemas para analizar la asignación de tiempo de los miembros de un hogar rural entre tareas productivas y reproductivas ver A. Deaton, 1997.

5 Traducción propia. 
de niños, y los cuidados de la salud. Stancanelli y Straton (2010) también trabajan con una definición de reproducción social que no incluye los servicios de cuidado, y solamente incluyen como trabajo del hogar las actividades rutinarias y obligatorias, tales como preparación de comida, limpieza, cuidado de la ropa, compras y pagos de servicios.

Las tareas de reproducción social al interior de un hogar pueden ser llevadas a cabo por los mismos miembros del hogar, por personas contratadas, o por ambas; en el caso de las personas contratadas, estas pueden o no vivir en el hogar. Si partimos de que la dotación de tiempo de los miembros del hogar puede ser dedicada a diversas actividades - el trabajo en el mercado, entre otras - en el caso en que el tiempo que los miembros del hogar están dispuestos a dedicar a las tareas del hogar sea menor al necesario para su reproducción, surgirá una demanda por trabajo del hogar remunerado.

Esta demanda puede tomar dos modalidades: trabajadoras que viven en el hogar demandante (cama adentro) y trabajadoras que vienen por horas a trabajar en el hogar demandante (cama afuera). Usualmente, las trabajadoras «cama adentro» realizan tareas múltiples y las trabajadoras "cama afuera» realizan una o dos tareas en los hogares que demandan sus servicios. Asimismo, es muy probable que las trabajadoras «cama adentro» también brinden cuidados no especializados a niños y adultos mayores en el hogar, tal como encuentra Mattingly (2012) en el caso de San Diego, California.

Siguiendo a Becker (1965), la economía del hogar estudia la manera como los miembros de una familia organizan su tiempo para producir bienes y servicios al interior del hogar a partir de una función de producción familiar donde los insumos son el tiempo de los miembros del hogar y bienes comprados en el mercado. Para obtener estos bienes de mercado se necesita dinero, lo cual lleva a que algunos miembros ofrezcan su fuerza laboral en el mercado de trabajo. Así, la decisión de asignación de la dotación de tiempo a las distintas actividades depende de la productividad relativa de dichos miembros para las tareas domésticas en relación al trabajo en el mercado. De acuerdo a esto es posible derivar funciones de oferta de trabajo basadas en estas diferencias, así como en la función de preferencias del hogar. Gronau (1977) va más lejos y analiza los efectos de un aumento de los salarios de mercado para las mujeres, y la diferencia en sus efectos sobre el tiempo que dedican a las tareas domésticas tomando en cuenta si están trabajando o no. Como sabemos, los cuestionamientos de Arrow (1966) sobre las funciones de utilidad familiar llevaron a Becker $(1974,1976)$ a proponer su modelo del «jefe de hogar dictador benevolente»; a partir de las críticas a este último modelo se generaron toda un familia de modelos de negociación cooperativa y no cooperativa - reseñados en Lundberg y Pollack (2007), Chiappori y Donni (2009) y C. Doss (2013) — en los cuales los miembros del hogar mantienen sus preferencias individuales sujetos a un conjunto presupuestal común. En todos estos modelos es posible incorporar la demanda de trabajo del hogar remunerado (THR) cuando el tiempo que los miembros de hogar están dispuestos a dedicar a dichas tareas es menor que el tiempo necesario para llevarlas a cabo. 
Stancanelli y Stratton (2010) analizan la demanda de trabajadoras del hogar en Inglaterra y Francia sobre la base de un modelo en el cual la función de bienestar familiar depende de las funciones de utilidad de cada miembro. A su vez, las funciones de utilidad individuales dependen del consumo privado y del ocio de cada uno, y de un bien público "producido» por medio de trabajo en el hogar. Las autoras maximizan la función de bienestar familiar sujeta a la función de producción del bien público, a las restricciones de tiempo de cada miembro del hogar y al conjunto presupuestal común. Encuentran que la probabilidad de emplear trabajo doméstico remunerado depende negativamente del precio de dicho trabajo y positivamente de los ingresos de cada miembro del hogar y del ingreso no laboral familiar ${ }^{6}$. Asimismo, encuentran que los efectos marginales del aumento de la tasa salarial de la mujer sobre la probabilidad de contratar trabajadoras del hogar son mayores que los efectos marginales del aumento de la tasa salarial de los varones. Así, una elevación de 1\% en los salarios de las mujeres lleva a un incremento de $10 \%$ en la probabilidad de contratar trabajo del hogar remunerado en Gran Bretańa, y de 7\% en el caso de Francia. Los porcentajes en el caso de los varones son de $8 \%$ y $2 \%$, respectivamente. Asimismo, las autoras encuentran que la contribución de los varones a las tareas domésticas aumenta a mayor sueldo de las mujeres (ver también Bloemen y Stancanelli, 2013).

Para el caso del Perú, García (2006) analiza la oferta de trabajo infantil sobre la base de un modelo donde la familia se compone del jefe de hogar, cónyuge e hijo. La función de utilidad familiar incluye un bien comprado en el mercado, un bien llamado «tareas del hogar» que es producido en casa, y la educación del hijo. En este modelo, las horas de trabajo del padre están dadas; la madre dedica su tiempo a las tareas del hogar y al mercado de trabajo; y el hijo añade a las actividades mencionadas, las horas de educación. Dado que la producción del bien «tareas del hogar» por parte de la madre y el hijo puede ser inferior a la necesaria, existe la posibilidad de contratar trabajo del hogar remunerado. Entonces, la familia maximiza la función de utilidad, sujeta a la función de producción del bien «tareas del hogar», las restricciones de tiempo de la madre y del hijo, y a la recta de presupuesto familiar. Con datos de la Encuesta Nacional de Niveles de Vida (ENNIV) para Perú, en los años 1997 y 20007, García (2007) estima la demanda por trabajo en el hogar y encuentra que depende negativamente de los salarios de mercado de la madre y del hijo, y que hay cierto grado de sustitución entre el tiempo de ambos. Asimismo, encuentra que el tiempo que los miembros de la familia dedican a las tareas del hogar depende de su sexo, su edad, la educación del jefe y las características del hogar. Si bien este trabajo no se centra en la demanda de trabajo del hogar remunerado,

\footnotetext{
6 Resultados similares obtienen Hersh y Stratton (1997) para Estados Unidos de Norteamérica.

7 El autor hace un pool con los datos de ambos ańos debido a que el porcentaje de nińos que trabajan y reciben un sueldo por ello es muy reducido en las encuestas individuales.
} 
las horas que la madre y el hijo dedican a tareas del hogar serían complementos de las horas de THR remunerado eventualmente demandadas.

Fakih y Marrouch (2012) trabajan con los datos de la Encuesta Nacional de Presupuesto de los Hogares 2005 llevado a cabo por la Administración Central de Estadísticas en Líbano. Los autores encuentran que la probabilidad de contratar THR es mayor en los hogares de mayor número de miembros, en aquellos donde el jefe de hogar es mujer, en las casas más grandes y en aquellos hogares con un mayor consumo agregado. Es necesario señalar que las trabajadoras del hogar son migrantes, ya que en Líbano no existe la costumbre de trabajar para otros hogares; esto hace que la mayoría de trabajadoras del hogar sean cama adentro. Adicionalmente, los autores encuentran que los hogares urbanos tienen una mayor probabilidad de retener a una trabajadora del hogar (migrante) que los hogares rurales.

Mattingly (2012) basa su análisis en entrevistas a trabajadoras del hogar y empleadores en San Diego, California. El objetivo de la autora es analizar la organización de los dos grupos de hogares, así como su interacción con relación a las tareas de cuidado. Así, encuentra que mientras que las empleadoras demandan THR para poder ofrecer su mano de obra al mercado, las trabajadoras del hogar se apoyan en familiares para las tareas de cuidado en sus propios hogares. Si bien estas tareas son básicamente de cuidado no especializado, no es aventurado esperar que lo mismo suceda con las tareas del hogar.

Klaveren, Brink y Praag (2011) examinan cómo la asignación del tiempo en las parejas y sobre todo sus horas de trabajo influyen en la demanda de trabajo del hogar y de cuidado de niños en Holanda. Encuentran que las parejas con hijos tienen menos superposición en sus horas de trabajo, y emplean más los cuidados informales que los formales, debido al alto costo de este último (unas 5 veces más caro). Los autores no encuentran un efecto del nivel de ingreso de los padres sobre la demanda de cuidado de sus hijos.

\section{LA DEMANDA DE TRABAJO DEL HOGAR REMUNERADO}

Nos basamos en los trabajos de Stancannelli y Straton (2010) y de García (2006, 2007), quienes trabajan con funciones de utilidad familiares. Si bien es cierto que los modelos de negociación cooperativa eficiente son más adecuados para analizar la interacción entre los miembros de una familia, lo que nos interesa analizar aquí es cómo se distribuye el tiempo de los miembros de la familia entre sus diversos usos, por lo cual asumiremos que ha habido una negociación previa entre los miembros del hogar con respecto al consumo individual del bien $C$ a producir. 
Por lo tanto si asumimos que los miembros del hogar que trabajan en el mercado no consumen tiempo libre, y que solamente consumen un bien hicksiano consistente en «el producto de las tareas del hogar» ${ }^{8}$, la función de utilidad familiar sería la siguiente:

$$
U=U(C)
$$

Para producir el bien $C$ se necesita tiempo de trabajo en el hogar y un insumo comprado en el mercado. Si $y$ representa dicho insumo, $d_{j}=1,2$ las horas dedicadas por los individuos al trabajo en el hogar, y $d_{T H R}$ las horas de THR demandadas, la función de producción de $C$ será":

$$
C=f\left(d_{1}, d_{2}, d_{T H R} ; y\right)
$$

El conjunto presupuestal del hogar será:

$$
\left(w_{1}+w_{2}\right) T+R=P_{C} C
$$

Donde $T$ es la dotación total de tiempo de cada miembro de la familia que trabaja, $R$ el ingreso no laboral del hogar $\mathrm{y} w_{j}, \forall j=1,2$ las tasas de salario respectivas. La restric-

ción de tiempo para cada individuo que trabaja será:

$$
T=d_{1}+l_{1}=d_{2}+l_{2}
$$

Siendo $\left(l_{1}, l_{2}\right)$ las horas de trabajo ofrecidas en el mercado por los miembros del hogar. Si reemplazamos la ecuación (2) en las expresiones (1) y (3), el problema económico a resolver es el siguiente:

$$
\begin{array}{ll}
\text { Max } & U=U\left[f\left(d_{1}, d_{2}, d_{T H R} ; y\right)\right] \\
\text { s.a. } & \left(w_{1}+w_{2}\right) T+R=P_{y} y+w_{1} d_{1}+w_{2} d_{2}+w_{T H R} d_{T H R} \\
& T=d_{1}+l_{1}=d_{2}+l_{2}
\end{array}
$$

A partir de la ecuación de Lagrange obtenemos las condiciones de óptimo del consumidor:

$$
\begin{aligned}
& \frac{\partial U}{\partial C} \frac{\partial f}{\partial d_{j}}=\lambda w_{j} \quad \forall j=1,2, T H R \\
& \frac{\partial U}{\partial C} \frac{\partial f}{\partial y}=\lambda P_{y}
\end{aligned}
$$

8 En caso de existir menores de edad en el hogar, estos no tendrían poder de decisión pero si acceso a ambos bienes debido al altruismo de los miembros que trabajan y tienen poder de decisión.

9 García $(2006,2007)$ emplea una función diferente, donde $C=f\left(d_{1}, d_{2}, y\right)+d_{T H R}$. 


$$
\frac{\partial \Lambda}{\partial \lambda}=\left(w_{1}+w_{2}\right) T+R-P_{y} y+w_{1}\left(d_{1}+h_{1}\right)+w_{2}\left(d_{2}+h_{2}\right)+w_{T H R} d_{T H R}=0
$$

La solución interior será la siguiente:

$$
\lambda=\frac{\left(\frac{\partial U}{\partial C}\right)\left(\frac{\partial f}{\partial d_{1}}\right)}{w_{1}}=\frac{\left(\frac{\partial U}{\partial C}\right)\left(\frac{\partial f}{\partial d_{2}}\right)}{w_{2}}=\frac{\left(\frac{\partial U}{\partial C}\right)\left(\frac{\partial f}{\partial d_{T H R}}\right)}{w_{T H R}}=\frac{\left(\frac{\partial U}{\partial C}\right)\left(\frac{\partial f}{\partial y}\right)}{P_{y}}
$$

Sobre la base de esta solución interior y de las restricciones del conjunto presupuestal familiar, obtenemos las funciones de demanda del insumo de mercado $(y)$, las funciones de demanda de las horas de trabajo en el hogar de sus miembros $\left(d_{1}, d_{2}\right)$, así como la función de demanda de trabajo del hogar remunerado $\left(d_{T H R}\right)$ :

$$
\begin{aligned}
& d_{y}=d_{y}\left(P_{y}, w_{1}, w_{2}, w_{T H R}, R\right) \\
& d_{j}=d_{j}\left(P_{y}, w_{1}, w_{2}, w_{T H R}, R\right) \quad \forall j=1,2, T H R
\end{aligned}
$$

Vemos así que la demanda de trabajo del hogar remunerado depende también del costo de oportunidad del tiempo de los miembros del hogar. Es decir, si la tasa de salarios de un miembro del hogar aumenta, y asumimos que el efecto sustitución es mayor que el efecto ingreso total ${ }^{10}$, este miembro del hogar trabajará más horas y dedicará menos tiempo a las tareas del hogar, lo cual aumentaría las horas dedicadas a trabajar por el otro miembro o por el trabajador del hogar remunerado.

Finalmente, a partir de las restricciones de tiempo de los miembros de la familia que trabajan, obtenemos sus funciones de oferta de trabajo al mercado laboral:

$$
l_{j}=T-d_{j}\left(P_{y}, w_{1}, w_{2}, w_{T H R}, R\right) \quad \forall j=1,2
$$

De tal manera que la oferta de trabajo al mercado de los miembros de la familia dependerá también del costo contratar trabajo del hogar remunerado. Con respecto a las soluciones de esquina, si $w_{1}{ }^{*}$ y $w_{2}{ }^{*}$ son los salarios de reserva de los miembros del hogar, y $w_{T H R}{ }^{*}$ es el precio de reserva del servicio "trabajo del hogar remunerado» ${ }^{11}$, hay cuatro posibles situaciones. En primer lugar tenemos el caso en el cual ambos miembros del hogar trabajan fuera del hogar, y además contratan THR:

$$
\begin{array}{llll}
w_{j}>w_{j}^{*} & \Rightarrow & l_{j}=l_{j}\left(P_{y}, w_{1}, w_{2}, w_{T H R}, R\right)>0 & \forall j=1,2 \\
w_{T H R}<w_{T H R} * \Rightarrow & d_{T H R}=d_{T H R}\left(P_{y}, w_{1}, w_{2}, w_{T H R}, R\right)>0 &
\end{array}
$$

\footnotetext{
${ }^{10}$ El efecto ingreso total se compone del efecto ingreso ordinario y el efecto ingreso dotación. El efecto neto depende de las magnitudes de ambos.

11 Servicio que es demandado por los miembros del hogar.
} 
En segundo lugar, el caso en que ambos miembros del hogar trabajan fuera del hogar, pero no contratan THR:

$$
\begin{array}{llll}
w_{j}>w_{j}^{*} & \Rightarrow & l_{j}=l_{j}\left(P_{y}, w_{1}, w_{2}, w_{T H R}, R\right)>0 \\
w_{T H R}>w_{T H R} * \Rightarrow & d_{T H R}=d_{T H R}\left(P_{y}, w_{1}, w_{2}, w_{T H R}, R\right)=0 &
\end{array} \quad \forall j=1,2
$$

En tercer lugar, el caso en que solo uno de los miembros del hogar trabaja fuera del hogar, y además se contrata THR:

$$
\begin{array}{lll}
w_{1}>w_{1} * & \Rightarrow & l_{1}=l_{1}\left(P_{y}, w_{1}, w_{2}, w_{T H R}, R\right)>0 \\
w_{1}<w_{1} * & \Rightarrow & l_{1}=l_{1}\left(P_{y}, w_{1}, w_{2}, w_{T H R}, R\right)=0 \\
w_{T H R}<w_{T H R} * & \Rightarrow & d_{T H R}=d_{T H R}\left(P_{y}, w_{1}, w_{2}, w_{T H R}, R\right)>0
\end{array}
$$

En cuarto lugar, el caso en que solo uno de los dos miembros del hogar trabaja, y no se contrata THR:

$$
\begin{array}{lll}
w_{1}>w_{1} * & \Rightarrow & l_{1}=l_{1}\left(P_{y}, w_{1}, w_{2}, w_{T H R}, R\right)>0 \\
w_{1}<w_{1}{ }^{*} & \Rightarrow & l_{1}=l_{1}\left(P_{y}, w_{1}, w_{2}, w_{T H R}, R\right)=0 \\
w_{T H R}>w_{T H R} * & \Rightarrow & d_{T H R}=d_{T H R}\left(P_{y}, w_{1}, w_{2}, w_{T H R}, R\right)=0
\end{array}
$$

En la siguiente sección analizamos los datos a partir de ambas encuestas, lo cual nos permitirá analizar las decisiones de demanda de THR en relación a la oferta de trabajo al mercado de los empleadores (miembros del hogar).

\section{LA DEMANDA DE TRABAJO DEL HOGAR REMUNERADO EN EL PERÚ}

En esta sección, empleamos los datos de la ENUT 2010 y de las ENAHO 2010 y 2016 para describir las características de los hogares urbanos que demandan trabajo del hogar remunerado (THR), y de aquellos que no lo hacen. Adicionalmente analizamos las horas que las trabajadoras del hogar dedican a cada tarea, así como la relación entre sus horas de THR y las horas de trabajo en el mercado del jefe de hogar y del cónyuge. A continuación estimamos la probabilidad de que un jefe de hogar contrate THR remunerado bajo las diferentes modalidades. Finalmente, estimamos la función de demanda de trabajo del hogar remunerado con los datos de las ENAHO 2010 y 2016.

La Encuesta Nacional de Uso del Tiempo (ENUT) del año 2010 fue llevada a cabo por el Ministerio de la Mujer y el Desarrollo Social (Mimdes) en colaboración con el Instituto Nacional de Estadística e Informática (INEI) ${ }^{12}$, a nivel nacional, urbano

12 Con asistencia técnica de la Comisión Económica para América Latina (Cepal). 
y rural, entrevistando a las personas de 12 años a más residentes habituales del hogar. La Encuesta Nacional de Hogares (ENAHO) es llevada a cabo por el Instituto Nacional de Estadística e Informática (INEI), a nivel nacional, urbano y rural, y se entrevista a las personas de 14 años a más que residen habitualmente en el hogar. El marco muestral de ambas encuestas se construyó a partir de los Censos Nacionales de los años 2005 y 2007.

\subsection{Características de los hogares demandantes}

En esta subsección analizamos las características de los hogares urbanos demandantes de trabajo del hogar remunerado (THR) bajo las modalidades cama adentro y cama afuera. Debemos señalar que si bien ninguna de las encuestas empleadas fue hecha para analizar la demanda de THR, la ENUT nos permite ver las características de los hogares que demandan el THR bajo las modalidades cama afuera y cama adentro; mientras que la ENAHO solamente nos permite hacer esto en el caso de los hogares que demandan THR «cama adentro». Asimismo, en el caso de la ENUT, existen preguntas sobre la utilización de otros tipos de ayuda doméstica remunerada y no remunerada, y es posible conocer las tareas que estas personas llevan a cabo en el hogar. En el caso de la ENAHO no tenemos información sobre las tareas del hogar, pero es posible asignar a las trabajadoras del hogar remuneradas bajo la modalidad cama adentro al hogar demandante, con lo cual podremos estimar la función de demanda de esta modalidad de THR. Asimismo, las ENAHO tienen los datos de las horas trabajadas por cada trabajadora y del salario que recibe, datos que no existen en la ENUT.

Trabajando primero con la ENUT, presentamos los promedios de horas que las trabajadoras del hogar remuneradas dedican a cada tarea en el sector urbano. Hemos reordenado los resultados de los módulos respectivos de manera que presentamos solamente las tareas que consideraremos como THR de acuerdo a la definición de la OIT. Es importante señalar que las preguntas son hechas al jefe de hogar o al cónyuge de las familias demandantes de THR, pero no es posible saber si las trabajadoras llevan a cabo todas estas actividades.

Son 391 hogares —alrededor de 672040 hogares con datos expandidos — los cuales demandan ayuda para llevar a cabo las tareas de hogar de personas que no viven en este, entre las cuales están las trabajadoras del hogar "cama afuera»" ${ }^{13}$. En el cuadro 1 vemos la lista de las tareas llevadas a cabo por estas trabajadoras Recordemos, que tal como dijimos en el párrafo anterior, no todas las trabajadoras del hogar llevan a cabo todas las tareas y no es posible obtener el dato de qué tareas realiza cada una. Es decir, la ENUT no nos permite identificar a cada trabajadora, si bien podemos identificar a los hogares

\footnotetext{
13 De acuerdo a la ENUT, existen hogares que demandan dos trabajadoras del hogar (4,2\% del total de hogares) o tres ( $0,7 \%$ del total). Dado el bajo porcentaje, en estos casos solamente tomaremos los datos de la primera trabajadora listada.
} 
que las contratan. Presentamos entonces los promedios de horas trabajadas durante la semana para cada tarea del hogar listada en el cuadro 1. Vemos así que la tarea del hogar que requiere más horas de trabajo en promedio es la preparación de comidas, y en segundo lugar la limpieza general de la vivienda, y luego viene el lavado y planchado de ropa. En el caso de las compras del hogar, en la muestra hay una persona a la que no se le paga por dicha tarea, lo cual puede ser un error de digitación.

Cuadro 1. Trabajo del hogar remunerado "cama afuera»

\begin{tabular}{lccc}
\hline & horas $\mathrm{L}-\mathrm{V}$ & horas $\mathrm{S}-\mathrm{D}$ & sí pagó \\
\hline Preparación de comidas & $6,7 \mathrm{~h}$ & $1,3 \mathrm{~h}$ & $100,0 \%$ \\
Lavado y barrido - cocina & $1,8 \mathrm{~h}$ & $0,3 \mathrm{~h}$ & $100,0 \%$ \\
Arreglo de habitaciones & $1,2 \mathrm{~h}$ & $0,3 \mathrm{~h}$ & $100,0 \%$ \\
Limpieza de cuarto de baño & $0,5 \mathrm{~h}$ & $0,5 \mathrm{~h}$ & $100,0 \%$ \\
Limpieza de la vivienda & $3,8 \mathrm{~h}$ & $0,7 \mathrm{~h}$ & $100,0 \%$ \\
Arreglo diario de la vivienda & $0,6 \mathrm{~h}$ & $0,1 \mathrm{~h}$ & $100,0 \%$ \\
Disposición de la basura & $0 \mathrm{~h}$ & $0,0 \mathrm{~h}$ & $100,0 \%$ \\
Acarreo de agua & $0,4 \mathrm{~h}$ & $0,2 \mathrm{~h}$ & $\mathrm{n}, \mathrm{a}$, \\
Lavado y planchado de ropa & $2,3 \mathrm{~h}$ & $0,7 \mathrm{~h}$ & $100,0 \%$ \\
Compras del hogar & $0,6 \mathrm{~h}$ & $0,1 \mathrm{~h}$ & $95,2 \%$ \\
\hline
\end{tabular}

Fuente: ENUT 2010. Elaboración propia.

En el Anexo 1 presentamos las tareas realizadas por las trabajadoras del hogar remuneradas bajo la modalidad cama afuera, por área geográfica (i.e. urbano y rural). Tal como esperábamos, la mayoría de trabajadoras del hogar se encuentran en el área urbana, pero lo interesante aquí es ver algunas diferencias de acuerdo al área geográfica. Vemos por ejemplo que tareas como el acarreo de agua es llevado a cabo por trabajadoras "cama afuera» en el caso del sector urbano, mas no en el sector rural. Luego de ver los datos sobre trabajo del hogar remunerado bajo la modalidad cama adentro y por área geográfica, tendremos una idea más clara de las divisiones de tareas entre los sectores urbano y rural.

En el cuadro 2 presentamos los datos de 47 trabajadoras del hogar remuneradas bajo la modalidad cama adentro - el número expandido es de alrededor de 96997 . Tal como en el caso del cuadro 1 hemos puesto solamente los promedios de horas por tarea, ya que no existe el dato por trabajadora. Vemos así que los promedios de horas de trabajo semanal por tarea son mayores en el cuadro 2 que en el cuadro 1 en la mayoría de los rubros. Un punto a notar es que el porcentaje de personas que reciben un pago por tarea no es siempre igual al 100\%, lo cual podría ser nuevamente un error de digitación de la encuesta. Examinando los datos encontramos que se trata de dos casos: una trabajadora de 27 ańos de edad, y otra de 63 ańos de edad. 
Cuadro 2. Trabajo del hogar remunerado cama adentro

\begin{tabular}{lccc}
\hline & horas L - V & horas S - D & le pagaron \\
\hline Preparación de comidas & $4,8 \mathrm{~h}$ & $1,3 \mathrm{~h}$ & $97,6 \%$ \\
Lavado y barrido - cocina & $0,7 \mathrm{~h}$ & $0,1 \mathrm{~h}$ & $95,7 \%$ \\
Arreglo de habitaciones & $0,7 \mathrm{~h}$ & $0,2 \mathrm{~h}$ & $97,4 \%$ \\
Limpieza de cuarto de baño & $0,3 \mathrm{~h}$ & $0,1 \mathrm{~h}$ & $97,6 \%$ \\
Limpieza de la vivienda & $2,8 \mathrm{~h}$ & $0,7 \mathrm{~h}$ & $97,5 \%$ \\
Arreglo diario de la vivienda & $0,2 \mathrm{~h}$ & $0,0 \mathrm{~h}$ & $97,1 \%$ \\
Disposición de la basura & $0,0 \mathrm{~h}$ & $0,0 \mathrm{~h}$ & $100,0 \%$ \\
Acarreo de agua & $0,0 \mathrm{~h}$ & $0,0 \mathrm{~h}$ & $100,0 \%$ \\
Lavado y planchado de ropa & $1,9 \mathrm{~h}$ & $0,7 \mathrm{~h}$ & $100,0 \%$ \\
Compras del hogar & $1,8 \mathrm{~h}$ & $0,6 \mathrm{~h}$ & $95,6 \%$ \\
\hline
\end{tabular}

Fuente: ENUT 2010. Elaboración propia.

En el Anexo 2 vemos que, si bien la mayoría de trabajadoras del hogar bajo la modalidad cama adentro, también se encuentran en el sector urbano, existen algunas diferencias con respecto a la modalidad "cama afuera». Por ejemplo, el porcentaje de trabajadoras del hogar "cama adentro» que se ocupa de la comida y de la limpieza en el sector rural es mayor que el porcentaje respectivo en el caso de las trabajadoras del hogar "cama afuera». Podríamos decir que en el área urbana la limpieza es llevada a cabo — cada vez máspor trabajadoras que no viven en el hogar.

En el cuadro 3 presentamos las características de los hogares urbanos que demandan trabajo del hogar remunerado (THR), bajo las modalidades cama adentro y cama afuera, así como de aquellos que no lo demandan, tanto con datos de la ENUT como de la ENAHO. En el caso de la ENUT 2010 no es posible «separar» a los hogares que solamente demandan THR «cama adentro» debido al diseńo de la encuesta, pero si ha sido posible separar a aquellos que solamente demandan THR «cama afuera» juntando el módulo de estas trabajadoras con el módulo de empleo e ingresos de los hogares demandantes. En el caso de las ENAHO 2010 y 2016, como ya dijimos, solamente es posible identificar a los hogares que demandan THR bajo la modalidad cama adentro, y a los que no lo demandan - entre los cuales algunos podrían estar demandando trabajo del hogar "cama afuera»" ${ }^{14}$.

Examinando los datos, constatamos que no hay una diferencia apreciable en el sexo del jefe de hogar por tipo de demanda de THR ${ }^{15}$. En cuanto al nivel de educación, vemos

14 De acuerdo a los datos de la ENAHO las trabajadoras del hogar «cama adentro» son el 14,2\% y el 7,2\% del total de trabajadoras del hogar para los años 2010 y 2016, respectivamente.

${ }^{15} \mathrm{Al}$ contrario de lo que señalan Fakih y Marrouch (2012) para el caso del Líbano; sin embargo, en dicho país las trabajadoras del hogar remuneradas son en su mayoría migrantes internacionales y no tendrían un hogar propio donde vivir. 
que los jefes de hogar que solamente contratan THR «cama adentro» tienen en su mayoría estudios universitarios, mientras que tanto aquellos que solamente contratan THR «cama afuera» como quienes no contratan trabajadoras del hogar tienen en su mayoría estudios secundarios. Esto es un indicador de los niveles de ingresos de los primeros, lo cual analizaremos a partir de los datos del cuadro 4. En el caso de los grupos de edad de los jefes de hogar, los cuales funcionan aquí como un proxy del ciclo de vida del hogar, vemos que los hogares relativamente más «jóvenes» demandan en su mayoría trabajo del hogar remunerado "cama afuera», lo cual estaría asociado a cambios generacionales en la organización de los hogares, tales como la mayor edad de la primera unión y la postergación de la maternidad. En cuanto a la lengua materna del jefe de hogar, si bien la mayoría habla el castellano, el porcentaje de jefes de hogar cuya lengua materna es el quechua es mayor entre quienes no demandan THR, que entre quienes lo demandan bajo alguna de sus modalidades.

Cuadro 3. Características de los hogares por modalidad de su demanda de THR

\begin{tabular}{|c|c|c|c|c|c|c|c|}
\hline & \multicolumn{3}{|c|}{ ENUT 2010} & \multicolumn{2}{|c|}{ ENAHO $2010^{* *}$} & \multicolumn{2}{|c|}{ ENAHO 2016** } \\
\hline & $\begin{array}{c}\text { THR } \\
\text { Caf }\end{array}$ & $\begin{array}{l}\text { THR } \\
\text { Cad* }\end{array}$ & Resto & $\begin{array}{c}\text { THR } \\
\text { Cad }\end{array}$ & Resto & $\begin{array}{l}\text { THR } \\
\text { Cad }\end{array}$ & Resto \\
\hline jefe de hogar varón & $70.4 \%$ & $79.1 \%$ & $74.0 \%$ & $77.1 \%$ & $76.3 \%$ & $70.4 \%$ & $73.8 \%$ \\
\hline \multicolumn{8}{|l|}{ nivel de educación - jefe de hogar } \\
\hline sin nivel / educación inicial & $4,6 \%$ & $0,0 \%$ & $3,8 \%$ & $0,1 \%$ & $3,5 \%$ & $0,3 \%$ & $2,8 \%$ \\
\hline primaria & $18,8 \%$ & $4,0 \%$ & $24,0 \%$ & $9,6 \%$ & $27,8 \%$ & $11,1 \%$ & $23,8 \%$ \\
\hline secundaria & $40,5 \%$ & $24,0 \%$ & $43,3 \%$ & $20,2 \%$ & $41,4 \%$ & $16,3 \%$ & $44,4 \%$ \\
\hline superior no universitaria & $17,5 \%$ & $1,3 \%$ & $16,7 \%$ & $15,4 \%$ & $13,4 \%$ & $7,1 \%$ & $13,6 \%$ \\
\hline superior universitaria & $17,3 \%$ & $54,8 \%$ & $11,6 \%$ & $34,1 \%$ & $12,0 \%$ & $35,5 \%$ & $13,5 \%$ \\
\hline posgrado & $1,3 \%$ & $15,9 \%$ & $0,6 \%$ & $12,6 \%$ & $1,9 \%$ & $29,7 \%$ & $1,9 \%$ \\
\hline \multicolumn{8}{|l|}{ grupos de edad del jefe de hogar } \\
\hline $15-24$ años & $3,4 \%$ & $2,6 \%$ & $4,5 \%$ & $0,0 \%$ & $1,7 \%$ & $0,0 \%$ & $1,6 \%$ \\
\hline $25-44$ años & $43,9 \%$ & $38,1 \%$ & $44,1 \%$ & $29,4 \%$ & $33,7 \%$ & $32,5 \%$ & $35,9 \%$ \\
\hline $45-64$ años & $31,5 \%$ & $29,6 \%$ & $38,9 \%$ & $40,7 \%$ & $47,4 \%$ & $28,5 \%$ & $46,5 \%$ \\
\hline 65 años y más & $21,2 \%$ & $29,7 \%$ & $12,5 \%$ & $29,9 \%$ & $17,2 \%$ & $39,0 \%$ & $16,0 \%$ \\
\hline \multicolumn{8}{|l|}{ lengua materna del jefe de hogar } \\
\hline quechua & $8,3 \%$ & $2,6 \%$ & $12,8 \%$ & $5,1 \%$ & $15,8 \%$ & $0,3 \%$ & $17,9 \%$ \\
\hline aymará & $2,6 \%$ & $0,0 \%$ & $2,3 \%$ & $0,3 \%$ & $2,1 \%$ & $\mathrm{n}, \mathrm{d}$ & $2,0 \%$ \\
\hline otra lengua nativa & $0,0 \%$ & $0,0 \%$ & $0,1 \%$ & $0,0 \%$ & $0,1 \%$ & $\mathrm{n}, \mathrm{d}$ & $0,2 \%$ \\
\hline castellano & $88,8 \%$ & $97,4 \%$ & $84,7 \%$ & $94,6 \%$ & $81,8 \%$ & $98,5 \%$ & $79,6 \%$ \\
\hline idioma extranjero & $0,3 \%$ & $0,0 \%$ & $0,1 \%$ & $\mathrm{n}, \mathrm{d}$ & $0,2 \%$ & $1,2 \%$ & $0,3 \%$ \\
\hline muestra & 391 & 43 & 2,573 & 891 & 37,459 & 1,200 & 59,811 \\
\hline datos expandidos & 672,040 & 79,036 & $4,588,101$ & 37,823 & $16,211,006$ & 441,548 & $22,012,954$ \\
\hline
\end{tabular}

Fuente: Elaboración propia sobre la base de los datos de la ENUT 2010 y las ENAHO 2010 y 2016.

* En algunos casos también demandan THR cama afuera.

** Tanto en THR Cad y en Resto es posible que además el hogar también contrate THR cama afuera. 
En el cuadro 4 presentamos los salarios promedio pagados a las trabajadoras del hogar, y las horas promedio de THR demandadas por los hogares. Asimismo, presentamos datos sobre las horas ofrecidas en el mercado de trabajo por el jefe de hogar y el cónyuge del hogar demandante, y sus ingresos laborales y no laborales. Debemos señalar que si bien la ENUT 2010 nos da una rica descripción de las actividades realizadas por la trabajadoras del hogar remuneradsa, no nos permite saber cuántas tareas realiza cada trabajadora; asimismo, las trabajadoras del hogar remuneradas no se encuentran en el módulo de empleo e ingresos, por lo cual no tenemos el dato de sus salarios. En el caso de las ENAHO 2010 y 2016 sí tenemos los salarios de las trabajadoras cama afuera y cama adentro, pero como ya dijimos antes, debido al diseńo de la encuesta, solamente es posible relacionar al segundo grupo con sus empleadores.

Una primera regularidad es que en los hogares que demandan THR, los cónyuges trabajan más horas que en aquellos que no demandan estos servicios, lo cual es consistente con la posibilidad de sustituir el trabajo del cónyuge en el hogar por el de las trabajadoras del hogar remuneradas. En el caso de la ENUT podemos ver que en los hogares que demandan THR «cama adentro» los cónyuges trabajan menos horas que en aquellos que demandan THR «cama afuera». Un segundo resultado es que los ingresos laborales por hora de los jefes de hogar y cónyuges son mayores en los hogares que contratan THR. Hubiera sido interesante comparar la relación entre los salarios de las trabajadoras del hogar bajo ambas modalidades con los ingresos laborales y no laborales del jefe del hogar y del cónyuge, pero el diseño de ambas encuestas no nos permite hacer la comparación. Aun así, hemos constatado que los hogares con mayores ingresos, sobre todo del cónyuge, son aquellos que demandan THR.

Cuadro 4. Horas de trabajo e ingresos laborales y no laborales - 2010 y 2016

\begin{tabular}{lcccccccc}
\hline & \multicolumn{3}{c}{ ENUT 2010 } & \multicolumn{2}{c}{ ENAHO 2010 } & \multicolumn{2}{c}{ ENAHO 2016 } \\
\cline { 2 - 8 } & $\begin{array}{c}\text { THR } \\
\text { Caf }\end{array}$ & $\begin{array}{c}\text { THR } \\
\text { Cad* }\end{array}$ & Resto & $\begin{array}{c}\text { THR } \\
\text { Cad }\end{array}$ & Resto** & $\begin{array}{c}\text { THR } \\
\text { Cad }\end{array}$ & Resto** \\
\hline horas de THR demandadas & n.d. & n.d. & 0,0 & 60,7 & 0,0 & 53,5 & 0,0 \\
horas de trabajo del jefe de hogar & 44,4 & 39,4 & 46,1 & 46,3 & 45,0 & 44,9 & 42,8 \\
horas de trabajo de la cónyuge & 35,9 & 40,2 & 33,4 & 38,9 & 35,3 & 41,5 & 32,8 \\
ingreso por hora de la trabajadora & n.d. & n.d. & n,a, & 3,8 & n,a, & 6,7 & n,a, \\
ingreso por hora del jefe de hogar & 2,9 & 7,3 & 1,7 & 32,6 & 10,3 & 47,1 & 13,0 \\
ingreso por hora de la cónyuge & 1,3 & 3,3 & 0,6 & 11,9 & 4,7 & 4,3 & 1,8 \\
ingreso no laboral del jefe & n.d. & n.d. & n.d. & $12,022,2$ & $3,433,1$ & $15,909,1$ & $3,745,2$ \\
ingreso no laboral de la cónyuge & n.d. & n.d. & n.d. & $3,406,8$ & 696,7 & $1,027,2$ & 313,5 \\
muestra & 391 & 43 & 2,573 & 891 & 37,459 & 1,200 & 59,811 \\
datos expandidos & 672,040 & 79,036 & $4,588,101$ & 37,823 & $16,211,006$ & 441,548 & $22,012,954$ \\
\hline
\end{tabular}

Fuente: Elaboración propia sobre la base de los datos de la ENUT 2010 y de las ENAHO 2010 y 2016.

* En algunos casos también demandan THR cama afuera.

** Tanto en THR Cad y en Resto es posible que además el hogar también contrate THR cama afuera. 


\subsection{ESTIMACIONES ECONOMÉTRICAS}

En esta sección vamos a estimar la probabilidad de contratar THR, ya sea "cama afuera» o "cama adentro", con los datos de la ENUT 2010 y de las ENAHO 2010 y 2016. Asimismo, vamos a estimar una función de demanda de THR «cama adentro» con los datos de las ENAHO 2010 y 2016. Como dijimos antes, el diseńo de la ENAHO no nos permite identificar a los empleadores de las trabajadoras del hogar "cama afuera» ${ }^{16}$.

\section{Probabilidad de contratar trabajadoras del hogar remuneradas}

En el caso de la estimación de contratar THR frente a no contratarlo, la ecuación general será la siguiente:

$$
\begin{aligned}
P=\alpha_{0} & +\alpha_{1} \text { JefeHogarVaron }+\alpha_{2} \text { LenguaNativa }+\alpha_{3} \ln \left(w_{1}\right)+\alpha_{4} \ln \left(w_{2}\right) \\
& +\alpha_{5} \ln \left(R_{1}\right)+\alpha_{6} \ln \left(R_{2}\right)+\alpha_{7} \text { NumHijosM } 6+u
\end{aligned}
$$

Donde $P$ será una variable de tres categorías si trabajamos con la ENUT, y dos categorías si trabajamos con la ENAHO. En el caso de la ENUT, dado que esta encuesta permite identificar a los hogares que contratan THR bajo ambas modalidades:

$$
\begin{array}{ll}
P=0 & \text { No contrata THR } \\
P=1 & \text { Contrata THR «cama adentro» } \\
P=2 & \text { Contrata THR «cama afuera» }
\end{array}
$$

Por lo tanto, estimamos la ecuación por medio de un Logit Multinomial. En el caso de las ENAHO solamente es posible identificar a los empleadores de las trabajadoras del hogar remuneradas "cama adentro», por lo cual $P$ será una variable dicotómica:

$$
\begin{array}{ll}
P=0 & \text { No contrata THR «cama adentro» } \\
P=1 & \text { Contrata THR «cama adentro» }
\end{array}
$$

Las variables exógenas en ambos casos serán JefeHogarVarón, variable dicotómica donde 1 representa al varón y 0 a la mujer; LenguaNativa, que es la lengua materna del jefe de hogar, variable dicotómica donde 1 representa una lengua materna nativa y 0 al resto de lenguas; el logaritmo natural de los ingresos laborales por hora del jefe de hogar $\left(w_{1}\right)$ y del cónyuge $\left(w_{2}\right)$, variables numéricas continuas; el logaritmo natural de los ingresos no laborales del jefe de hogar $\left(R_{1}\right)$ y del cónyuge $\left(R_{2}\right)$, también variables numéricas continuas y disponibles solamente en el caso de las ENAHO; y el número de hijos menores de 6 ańos de edad (NumHijosM6). Adicionalmente estimaremos ecuaciones

\footnotetext{
${ }^{16}$ Así como no nos permite estimar la oferta de trabajo de las trabajadoras «cama adentro» ya que no tenemos todos sus datos familiares. La estimación de la oferta de trabajo del hogar remunerado hecha en Garavito (2017) corresponde a las trabajadoras del hogar «cama afuera».
} 
Logit Binomiales con los datos de la ENUT y la ENAHO 2010, con las variables comunes de ambas encuestas.

En el cuadro 5a presentamos los resultados de la estimación de Logit Multinomial con los datos de la ENUT 2010, donde estimamos la probabilidad de contratar THR «cama adentro» y THR "cama afuera», en relación a no contratarlo. Así vemos que si el jefe de hogar es varón, la probabilidad de contratar THR en general es menor que cuando es mujer, lo cual es consistente con el hecho que la mayoría de mujeres jefas de hogar trabajan y no tienen un cónyuge que apoye en dichas tareas. La probabilidad de contratar THR también es menor si el jefe tiene una lengua materna nativa; si bien en el Perú la lengua materna no es un buen indicador de etnicidad, si está asociada a un menor conocimiento del mercado laboral urbano y a discriminación salarial y ocupacional. Los ingresos laborales del jefe y del cónyuge aumentan la probabilidad de contratar THR, siendo los efectos marginales mayores en el caso del THR «cama afuera», y en esta modalidad el efecto marginal del ingreso del cónyuge es mayor que el del jefe de hogar. Finalmente, la presencia de hijos menores de 6 años aumenta la probabilidad de contratar THR bajo ambas modalidades, en relación a no contratarlo. Sin embargo, debemos tomar en cuenta que en el caso de la ENUT no hemos tomado en cuenta las tareas de cuidado de niños en nuestra definición de THR. Por lo tanto, la presencia de niños menores tendría un efecto sobre la contratación de trabajadoras del hogar para realizar las otras tareas especificadas.

Cuadro 5a. Probabilidad de contratar THR - ENUT 2010

\begin{tabular}{|c|c|c|}
\hline Logit multinomial (2010) & \multirow{2}{*}{ contrata THR Caf } & \multirow{2}{*}{ contrata THR Cad } \\
\hline Base $=$ no contrata THR & & \\
\hline jefe de hogar varón & $-0,3159$ & $-1,6709^{* *}$ \\
\hline lengua materna del jefe & $-1,2083^{* *}$ & $-14,3397$ \\
\hline ln(ingreso horario jefe) & $0,3199^{* * *}$ & $17877^{* * *}$ \\
\hline $\ln ($ ingreso horario conyuge) & $0,4064^{* * *}$ & 0,2934 \\
\hline número de hijos menores de 6 años & $0,2278^{*}$ & 0,5549 \\
\hline constante & $-2,1104^{* * *}$ & $-6,7951^{* * *}$ \\
\hline \multicolumn{3}{|l|}{ Efectos marginales } \\
\hline jefe de hogar varón & $-0,0340$ & $-0,0009$ \\
\hline lengua materna del jefe & $-0,0868^{* * *}$ & $-0,0017$ \\
\hline $\ln$ (ingreso horario jefe) & $0,0321^{* *}$ & 0,0005 \\
\hline $\ln$ (ingreso horario conyuge) & $0,0409^{* * *}$ & 0,0001 \\
\hline número de hijos menores de 6 años & $0,0229^{*}$ & 0,0002 \\
\hline LR Chi 2 (10) & \multicolumn{2}{|c|}{$92.66^{* * *}$} \\
\hline Muestra & \multicolumn{2}{|c|}{1,135} \\
\hline
\end{tabular}


En el cuadro 5b con los datos de las ENAHO 2010 y 2016 podemos ver que si el jefe de hogar tiene una lengua materna nativa, la probabilidad de que contrate THR «cama adentro» con relación a no contratarlo es menor que si su lengua es el castellano o una lengua extranjera. Sin embargo el efecto marginal en valor absoluto es menor en el año 2016 que en el año 2010. Los ingresos laborales por hora del jefe de hogar y del cónyuge aumentan la probabilidad de contratar THR «cama adentro», si bien los efectos marginales de los ingresos laborales del jefe son mayores que los del ingreso laboral del cónyuge. Como vimos en la sección descriptiva de los datos, son los hogares con mayor edad promedio y mayores ingresos los que contratan THR «cama adentro» en su mayoría, lo cual es consistente con jefes de hogar que tienen un ingreso laboral por hora mayor que el cónyuge. En cuanto a los ingresos no laborales, estos representan también el poder de negociación de ambos cónyuges, además de la existencia de mayores ingresos. Todos los coeficientes son positivos, y en este caso los efectos marginales de los ingresos no laborales de los cónyuges son mayores que los de los jefes de hogar. Finalmente el número de hijos menores que seis años tiene un efecto positivo sobre la contratación de trabajadoras del hogar remuneradas bajo la modalidad cama adentro, como vimos también en el cuadro 5a.

Cuadro 5b. Probabilidad de contratar THR - ENAHO 2010 y 2016

\begin{tabular}{lcc}
\hline \multicolumn{1}{c}{ Logit binomial } & 2010 & 2016 \\
\hline Base=no contrata THR Cad & contrata THR CAd & contrata THR CAd \\
\hline jefe de hogar varón & 0,1066 & $-0,2897$ \\
lengua materna del jefe & $-1,1688^{* *}$ & $-1,3681^{*}$ \\
ln(ingreso horario jefe) & $0,6078^{* * *}$ & $0,9104^{* * *}$ \\
ln(ingreso horario conyuge) & $0,3836^{* * *}$ & $0,4873^{* * *}$ \\
ln(ingreso no laboral del jefe) & 0,0462 & 0,0370 \\
ln(ingreso no laboral del cónyuge) & $0,1113^{* *}$ & 0,0574 \\
número de hijos menores de 6 años & $0,6676^{* *}$ & 0,0963 \\
constante & $-6,9908^{* * *}$ & $-8,4813^{* * *}$ \\
\hline Efectos marginales & & \\
\hline jefe de hogar varón & 0,0005 & $-0,0004$ \\
lengua materna del jefe & $-0,0047^{* *}$ & $-0,0011^{* *}$ \\
$\ln ($ ingreso horario jefe) & $0,0032^{* * *}$ & $0,0010^{* * *}$ \\
$\ln ($ ingreso horario conyuge) & $0,0019^{* *}$ & $0,0006^{* *}$ \\
$\ln ($ ingreso no laboral del jefe) & 0,0002 & 0,00004 \\
$\ln ($ ingreso no laboral del cónyuge) & $0,0006^{* *}$ & 0,00006 \\
número de hijos menores de 6 años & $0,0035^{* *}$ & 0,0001 \\
\hline LR Chi 2 (7) & $120,70^{* * *}$ & $115,17^{* * *}$ \\
Muestra & 5,146 & 20,396 \\
\hline
\end{tabular}

Fuente de datos: ENUT 2010; ENAHO 2010 y 2016

*** significativo al $1 \%$

** significativo al $5 \%$

* significativo al $10 \%$ 
En el cuadro $5 \mathrm{c}$ presentamos los resultados de estimaciones Logit Binomiales con datos de la ENUT y de la ENAHO 2010. En estas regresiones estimamos la probabilidad de contratar THR «cama adentro» en relación a no contratarlo, con las variables comunes de ambas encuestas. El primer resultado que llama la atención es que con los datos de la ENAHO existe mayor probabilidad de que un jefe de hogar varón contrate THR «cama adentro", resultado que si bien estaba también en el cuadro 5b, no era significativo. Es además un resultado contrario al signo encontrado con la ENUT, pero debemos recordar que en el caso de la ENAHO no ha sido posible separar a los empleadores de THR «cama afuera», por lo cual nuestra comparación de resultados es bastante gruesa. No hay mayores diferencias en los signos de las demás variables frente a las estimaciones hechas en los Cuadros $5 \mathrm{a}$ y $5 \mathrm{~b}$.

Cuadro 5c. Comparación resultados 2010 ENUT y ENAHO

\begin{tabular}{lcc}
\hline \multicolumn{1}{c}{ Logit binomial (2010) } & $\mathbf{2 0 1 0}$ & $\mathbf{2 0 1 0}$ \\
\hline Base=no contrata THR Cad & contrata THR CAd & contrata THR CAd \\
\hline jefe de hogar varón & $-0,2794$ & $0,6853^{* *}$ \\
lengua materna del jefe & $-1,1896^{* *}$ & $-1,2834^{* *}$ \\
ln(ingreso horario jefe) & $0,2908^{* * *}$ & $0,6892^{* * *}$ \\
ln(ingreso horario conyuge) & $0,3840^{* * *}$ & $0,4229^{* * *}$ \\
número de hijos menores de 6 ańos & 0,2038 & $0,7329^{* * *}$ \\
constante & $-2,1091^{* * *}$ & $-7,2652^{* * *}$ \\
\hline Efectos marginales & & \\
\hline jefe de hogar varón & $-0,0298$ & $0,0028^{* * *}$ \\
lengua materna del jefe & $-0,0855^{* * *}$ & $-0,0044^{* * *}$ \\
ln(ingreso horario jefe) & $0,0290^{* * *}$ & $0,0031^{* * *}$ \\
ln(ingreso horario conyuge) & $0,0384^{* * *}$ & $0,0019^{* * *}$ \\
número de hijos menores de 6 años & 0,0204 & $0,0033^{* * *}$ \\
\hline LR Chi 2 & $50,69^{* * *}$ & $134,83^{* * *}$ \\
Muestra & 1,135 & 7,696 \\
\hline
\end{tabular}

Fuente de datos: ENUT 2010; ENAHO 2010 y 2016

*** significativo al $1 \%$

** significativo al $5 \%$

* significativo al $10 \%$

\section{Estimación de la función de demanda de THR «cama adentro»}

Como se discutió en las secciones anteriores, solamente podemos estimar una función de demanda de THR en el caso de las ENAHO y solamente para las trabajadoras que laboran bajo la modalidad cama adentro. La ecuación a estimar será la siguiente:

$$
\begin{aligned}
& \ln \left(h_{\text {THR }}^{d}\right)=\beta_{0}+\beta_{1} \text { JefeHogarVaron }+\beta_{2} \ln \left(w_{T H R}\right)+\beta_{3} \ln \left(w_{1}\right)+ \\
& \beta_{4} \ln \left(w_{2}\right)+\beta_{5} \ln \left(R_{1}\right)+\beta_{6} \ln \left(R_{2}\right)+\beta_{7} \text { NumMujeresHogar }+\beta_{8} L M+u
\end{aligned}
$$


Donde $h_{T H R}^{d}$ son las horas de trabajo del hogar remuneradas cama adentro. Además de las variables de ingresos laborales y no laborales del jefe de hogar y del cónyuge, incluimos la variable dicotómica LM, la cual indica si el hogar demandante está en Lima Metropolitana, la capital del Perú y el mercado más grande del país. Una variable de control adicional que apunta a captar una posible sustituibilidad del THR y el trabajo del hogar de los miembros del hogar es el número de mujeres en el hogar ${ }^{17}$. Un determinante importante de las horas de trabajo del hogar demandadas es la tasa salarial de la trabajadora del hogar $\left(w_{T H R}\right)$. Sin embargo, dado que esta variable puede estar correlacionada con el término de error, es necesario buscar un instrumento que no tenga ese problema. Por otro lado, dado que la muestra está truncada debido a que no todas las familias demandan THR es necesario estimar la función de demanda de THR empleando el método de selección de Heckman ${ }^{18}$. La ecuación de selección empleada es la siguiente:

$$
\begin{aligned}
P=\gamma_{0} & +\gamma_{1} \text { JefeHogarVaron }+\gamma_{2} \text { EdadJefe }+\gamma_{3} \ln \left(R_{1}\right)+\gamma_{4} \ln \left(R_{2}\right) \\
& +\gamma_{5} \text { LenguaNativa }+\gamma_{6} \text { NumHijosM } 6+u
\end{aligned}
$$

Donde P es igual a 1 si el jefe de hogar demanda THR «cama adentro» y 0 en caso contrario. Las variables de control incluyen ahora la edad del jefe de hogar como una proxy del ciclo de vida del hogar demandante.

En el cuadro 6 presentamos todas las estimaciones realizadas, tomando en cuenta los elementos señalados arriba. En primer lugar, en las columnas 1 y 3 estimamos la función de demanda de THR por el método de Heckman, sin tomar en cuenta la endogeneidad de la tasa salarial de la trabajadora del hogar ${ }^{19}$. Un primer resultado es que el lambda de Mills tiene un coeficiente significativo solamente en el año 2010, por lo cual estimamos nuevamente la demanda de THR para el año 2016 por medio del método de Mínimos Cuadrados Ordinarios (MCO) y presentamos los resultados en la columna 4. Entonces, comparando las columnas 1 y 4 podemos ver que si el jefe de hogar es varón, la cantidad de horas de THR demandas será mayor, lo cual podría explicarse por el mayor tamaño del hogar. La cantidad demandada de THR es menor a mayor tasa de salarios de la trabajadora, aun cuando empleamos variables instrumentales (columnas 2 y 5 ). Encontramos asimismo que a mayores ingresos del jefe de hogar y del cónyuge, mayores son las horas demandadas, lo cual implica un efecto sustitución de su trabajo las tareas de hogar por el de la trabajadora del hogar remunerada. En cuanto al número de mujeres en el hogar, este efectivamente reduce las horas de THR demandadas, tal como sucedería en los

\footnotetext{
17 En una visión tradicional de la familia las mujeres son las que llevan a cabo las tareas del hogar y no los varones; debido a que es posible que algunas generaciones mayores aún tengan sus hogares organizados de esta manera, ponemos esta variable de control.

18 Una limitación de este trabajo es que no eliminamos la heterogeneidad no explicada. Para ver cómo es posible hacerlo con un panel y empleando también la corrección por sesgo de Heckman ver Kyriazidou, 1997. 19 Tratamos de estimar su salario por medio de algunas variables de control como su educación, su edad y su condición de migrante, pero el bajo número nos dio coeficientes no significativos.
} 
hogares tradicionales. Finalmente, las jornadas laborales serán más largas si el empleador vive en Lima Metropolitana. En este punto es necesario discutir la jornada de trabajo de una trabajadora del hogar "cama adentro", ya que esta no tiene mayor control sobre las horas que trabaja si vive dentro del hogar de su empleador. Sin embargo, si pueden variar las horas que se requiere su trabajo, reduciendo sus períodos de descanso de acuerdo a las necesidades de los empleadores ${ }^{20}$.

En las columnas 2 y 5 estimamos las ecuaciones de demanda de trabajo del hogar remunerado por el método de variables instrumentales, donde empleamos tres instrumentos: el nivel de educación de la trabajadora, su edad y su condición de migrante a la vez. En versiones iniciales de este trabajo probamos con la educación y la edad de la trabajadora, como variables por separado y combinadas, pero el coeficiente no fue significativo, lo cual cambió al agregar la condición de migrante (por distrito) de la trabajadora. Así vemos que los efectos son negativos y significativos para ambos años, y muy parecidos. El hecho que las elasticidades de demanda de horas de trabajo - tasa de salario estén alrededor de - 0.41 nos indica que este es un servicio considerado básico por la población que lo demanda. De nuevo, la población con mayores ingresos y de mayor edad.

Por lo tanto, podemos decir que la demanda de horas de THR «cama adentro» tiene una relación negativa con la tasa salarial pagada a las trabajadoras y con el número de mujeres en el hogar, y positiva con los ingresos del jefe de hogar y del cónyuge. Asimismo, las jornadas laborales serán más largas en Lima Metropolitana que en el resto del Perú urbano.

Cuadro 6. Estimación de la demanda de THR cama adentro

\begin{tabular}{lccccc}
\hline \multirow{2}{*}{$\ln ($ horas trabajadas) } & \multicolumn{3}{c}{2010} & \multicolumn{3}{c}{2016} \\
\cline { 2 - 6 } & Inwthr & lnwthr=VI & Inwthr & Inwthr & Inwthr=VI \\
\hline jefe de hogar varón & $0,0788^{* *}$ & $0,1194^{* * *}$ & 0,0118 & 0,0074 & 0,0029 \\
ln (wthr) & $-0,3572^{* * *}$ & $-0,4252^{* * *}$ & $-0,4215^{* * *}$ & $-0,4199^{* * *}$ & $-0,4087^{* * *}$ \\
ln(ingresohorario jefe) & 0,0102 & 0,0013 & 0,0137 & 0,0116 & 0,0121 \\
ln(ingresohorario cónyuge) & 0,0085 & $-0,0098$ & $0,0363^{* *}$ & $0,0345^{* *}$ & $0,0345^{* *}$ \\
número de mujeres en el hogar & $-0,0084$ & 0,0000 & $-0,0338^{* *}$ & $-0,0329^{*}$ & $-0,0321^{*}$ \\
Lima Metropolitana & 0,1686 & $0,1947^{* * *}$ & $0,2469^{* * *}$ & $0,2456^{* * *}$ & $0,2359^{* *}$ \\
constante & $3,8251^{* * *}$ & $4,3212^{* * *}$ & $4,4503^{* * *}$ & $4,5766^{* * *}$ & $4,5597^{* * *}$ \\
\hline Select & & & & & \\
\hline jefe de hogar varón & $-0,0085^{* * *}$ & & 0,0265 & & \\
edad del jefe de hogar & $0,0396^{* * *}$ & & $-0,00002$ & & \\
ln(ingreso no laboral jefe) & $0,0459^{* * *}$ & & $0,0375^{* * *}$ & & \\
ln (ingreso no laboral cónyuge) & $0,0469^{* * *}$ & & $0,0422^{* * *}$ & & \\
lengua nativa del jefe de hogar & $-0,5796^{* * *}$ & & $-0,4611^{* * *}$ & & \\
número de hijos menores de 6 ańos & $0,1407^{* * *}$ & & $0,1335^{* *}$ & & \\
constante & $-2,2687^{* * *}$ & $-3,0099^{* * *}$ & & \\
\hline
\end{tabular}

20 Obviamente esto atenta contra sus derechos laborales. Ver Garavito, 2015. 


\begin{tabular}{lccccc}
\hline \multirow{2}{*}{$\ln ($ horas trabajadas) } & \multicolumn{2}{c}{2010} & \multicolumn{2}{c}{2016} & \\
\cline { 2 - 6 } & Inwthr & lnwthr=VI & Inwthr & Inwthr & Inwthr=VI \\
\hline Lambda de Mills & $0,1645^{* * *}$ & & 0,0406 & & \\
\hline número de observaciones & 59,776 & 458 & 69,379 & 220 & 220 \\
observaciones censuradas & 59,318 & & 69,159 & & \\
observaciones no censuradas & 458 & & 220 & & \\
R2 & & 0,3424 & & 0,4568 & 0,4666 \\
F & & & & $29,86^{* * *}$ & \\
Test de Breusch - Pagan Chi2(1) & & & & 0,00 & \\
Test de Wald Chi2(6) & $268,46^{* * *}$ & $44,46^{* * *}$ & $184,99^{* * *}$ & & $50,19^{* * *}$ \\
rho & 0,6222 & & 0,1886 & & \\
sigma & 0,2643 & & 0,2154 & & \\
\hline
\end{tabular}

Fuente: Estimación propia sobre la base de los datos de las ENAHO-INEI

*** significativo al $\left.1 \%\right|^{* *}$ significativo al 5\% ${ }^{*}$ significativo al $10 \% \mid \mathrm{VI}=($ educación, edad y cond. migrante de la trabajadora)

\section{CONCLUSIONES}

El análisis del mercado de trabajo del hogar remunerado es necesario para mejorar la situación de este grupo de trabajadoras. Los cambios en este mercado a lo largo del tiempo, desde la migración de mujeres y nińas del campo hacia la ciudad para trabajar en condiciones vulnerables a comienzos del siglo XX, hasta las relaciones más o menos reguladas de la actualidad, no han alterado la esencia jerárquica de esta relación y la dificultad de su regulación para asegurar el cumplimiento de normas mínimas de trabajo. Las trabajadoras del hogar remuneradas continúan siendo un grupo con bajos niveles de educación y pocas posibilidades de mejorar su situación, aun cuando las relaciones entre estas y sus empleadores parecieran dirigirse —al menos en el papel— hacia una relación de mercado.

En este trabajo hemos analizado la demanda de trabajo del hogar remunerado, en sus modalidades cama adentro y cama afuera, así como su relación con la organización del hogar de los empleadores, con los datos de la Encuesta Nacional de Uso del Tiempo para el año 2010 y de la Encuesta Nacional del Hogares, para los años 2010 y 2016.

Encontramos en primer lugar que los hogares que demandan trabajo del hogar remunerado bajo la modalidad cama dentro son aquellos con mayores niveles de educación y de ingresos, y mayores edades promedio, lo cual implica un hogar ya establecido. Los jóvenes demandan en su mayor parte trabajo del hogar remunerado bajo la modalidad cama afuera, lo cual tiene que ver con sus menores ingresos, retraso en la maternidad - o decisión de no tener hijos- y con la manera distinta en que organizan sus hogares.

Con los datos de la Encuesta Nacional de Uso del Tiempo (ENUT) para el año 2010 pudimos estimar la probabilidad de contratar THR bajo ambas modalidades, en relación a no contratarlo. Encontramos que aquellos jefes de hogar cuya lengua materna 
es nativa (quechua, aymará o una de las lenguas amazónicas) tienen una menor probabilidad de contratar THR en general. Asimismo, los ingresos laborales del jefe de hogar y del cónyuge aumentan la probabilidad de contratar THR, siendo el efecto marginal del ingreso laboral del cónyuge mayor que el del ingreso laboral del jefe de hogar para el caso del THR «cama afuera». En el caso del THR «cama adentro» se da lo contrario, como vemos también con los datos de la Encuesta nacional de Hogares (ENAHO). Como dijimos antes, es muy posible que los hogares «mayores» se organicen de la manera tradicional, y contraten THR «cama adentro» dependiendo más de los ingresos del jefe varón. Finalmente, el número de hijos menores de 6 años, que es la edad a la cual los niños entran al sistema escolar primario, aumenta la probabilidad de contratar trabajadoras del hogar.

Trabajando con los datos de la ENAHO, que nos permiten estimar la probabilidad de contratar THR «cama adentro» en relación a no contratarlo, vemos de nuevo que si bien los ingresos laborales por hora del jefe de hogar y del cónyuge aumentan la probabilidad de contratar trabajadoras del hogar, el efecto marginal del ingreso laboral del jefe es mayor que el del ingreso laboral de la cónyuge, tal como encontramos con la ENUT. En estas estimaciones incluimos también los ingresos no laborales del jefe y del cónyuge, no disponibles en la ENUT, y encontramos que los ingresos no laborales del cónyuge tienen un efecto positivo sobre la probabilidad de contratar THR "cama adentro", siendo los efectos marginales mayores que en el caso del jefe de hogar. Encontramos el mismo efecto positivo de la presencia de hijos menores de seis años sobre la probabilidad de contratar THR «cama adentro».

Por lo tanto, podemos decir que si bien la contratación de trabajo del hogar remunerado "cama afuera» se está reduciendo en relación al THR «cama afuera», este persiste en los hogares «mayores» y con más ingresos, mientras los hogares más jóvenes contratan en su mayoría trabajadoras del hogar por horas. Asimismo, podemos decir que los ingresos laborales y no laborales llevan a una mayor probabilidad ce contratar THR en relación a no hacerlo, y que los efectos marginales del ingreso laboral del cónyuge son mayores para la modalidad "cama adentro", sucediendo lo contrario para la modalidad "cama afuera».

Estimamos asimismo la demanda de trabajo del hogar remunerado bajo la modalidad cama adentro con los datos de la ENAHO para los ańos 2010 y 2016. Encontramos que las horas de THR que los empleadores demandan dependen negativamente de la tasa de salarios pagada a la trabajadora del hogar. Asimismo, existe un efecto sustitución entre el trabajo que los miembros del hogar puedan realizar en este y el que la trabajadora del hogar realiza. Al elevarse los ingresos salariales del jefe de hogar y del cónyuge, se hace más barato — relativamente - sustituir el trabajo en el hogar de sus miembros por trabajo del hogar contratado. Se da asimismo un efecto ingreso que lleva a demandar más horas de un servicio cuya demanda es relativamente inelástica. Encontramos asimismo, que la presencia de mujeres en el hogar reduce las horas demandadas de THR «cama adentro» con lo cual podemos decir que los hogares que demandan estos servicios 
todavía están organizados de manera tradicional. Finalmente, las jornadas laborales son mayores si el empleador vive en Lima Metropolitana, lo cual está relacionado a que este es el mayor mercado de trabajo del hogar remunerado en el Perú, donde también se encuentra la mayor oferta.

Anexo 1. Trabajado del hogar remunerado cama afuera por área geográfica

\begin{tabular}{lccc}
\hline & Urbano & Rural & Perú \\
\hline Preparación de comidas & $91.8 \%$ & $8.2 \%$ & $100.0 \%$ \\
Lavado y barrido - cocina & $91.7 \%$ & $8.3 \%$ & $100.0 \%$ \\
Arreglo de habitaciones & $87.1 \%$ & $12.9 \%$ & $100.0 \%$ \\
Limpieza de cuarto de baño & $93.0 \%$ & $7.0 \%$ & $100.0 \%$ \\
Limpieza de la vivienda & $92.0 \%$ & $8.0 \%$ & $100.0 \%$ \\
Arreglo diario de la vivienda & $92.5 \%$ & $7.5 \%$ & $100.0 \%$ \\
Disposición de la basura & $91.4 \%$ & $8.6 \%$ & $100.0 \%$ \\
Acarreo de agua & $100.0 \%$ & $0.0 \%$ & $100.0 \%$ \\
Lavado y planchado de ropa & $96.1 \%$ & $3.9 \%$ & $100.0 \%$ \\
Compras del hogar & $91.3 \%$ & $8.7 \%$ & $100.0 \%$ \\
\hline
\end{tabular}

Fuente: ENUT 2010. Elaboración propia.

Anexo 2. Trabajo del hogar remunerado cama adentro por área geográfica

\begin{tabular}{lccc}
\hline & Urbano & Rural & Perú \\
\hline Preparación de comidas & $90.0 \%$ & $10.0 \%$ & $100.0 \%$ \\
Lavado y barrido - cocina & $82.5 \%$ & $17.5 \%$ & $100.0 \%$ \\
Arreglo de habitaciones & $92.2 \%$ & $7.8 \%$ & $100.0 \%$ \\
Limpieza de cuarto de baño & $92.9 \%$ & $7.1 \%$ & $100.0 \%$ \\
Limpieza de la vivienda & $89.3 \%$ & $10.7 \%$ & $100.0 \%$ \\
Arreglo diario de la vivienda & $93.7 \%$ & $6.3 \%$ & $100.0 \%$ \\
Disposición de la basura & $92.2 \%$ & $7.8 \%$ & $100.0 \%$ \\
Acarreo de agua & $70.6 \%$ & $29.4 \%$ & $100.0 \%$ \\
Lavado y planchado de ropa & $90.4 \%$ & $9.6 \%$ & $100.0 \%$ \\
Compras del hogar & $91.3 \%$ & $8.7 \%$ & $96.7 \%$ \\
\hline
\end{tabular}

Fuente: ENUT 2010. Elaboración propia. 


\section{REFERENCIAS BIBLIOGRÁFICAS}

Arrow, Kenneth (1966). Social Choice and Individual Values. Third Edition. New York: John Wiley \& Sons.

Becker, Gary (1965). A theory of the allocation of time. Economic Journal, 75(299), September. https://doi.org/10.2307/2228949

Becker, Gary (1974). A Theory of Social Interactions. Journal of Political Economy, 82(6). 1063-1091. https://doi.org/10.1086/260265

Becker, Gary (1976). Altruism, Egoism, and Genetic Fitness: Economics and Sociobiology. Journal of Economic Literature, 14(3), 817-826.

Bloemen, Hans y Elena G.F. Stancanelli (2013). Market hours, household work, child care, and wage rates of partners: an empirical analysis. University of Amsterdam \& Paris School of Economics. https://doi.org/10.1007/s11150-013-9219-4

Blofield, Merike (2009). Feudal Enclaves and Political Reforms: Domestic Workers in Latin America. Latin American Research Review, 44(1), 158-190. https://doi.org/10.1353/ lar.0.0068

Chiappori, Pierre-André y Olivier Donni (2009). Non-unitary Models of Household Behavior: A Survey of the Literature. Discussion Paper No 4603, Institute for the Study of Labor.

Consejo de Ministras de la Mujer de Centroamérica (COMMCA) (2010). La institucionalización sociocultural y juridica de la desigualdad: el trabajo doméstico remunerado. Resúmenes de estudios de la región de Centroamérica y República Dominicana. San Salvador: Secretaría Jurídica del Sistema de la Integración Centroamericana.

Cortés, Patricia y Jessica Pan (2013). Outsourcing Household Production: Foreign Domestic Workers and Native Labor Supply in Hong Kong. Journal of Labor Economics, 31(2), 327-371. https://doi.org/10.1086/668675

Cox, Rosie (2000). Exploring the Growth of Paid Domestic Labour: A Case Study of London. Geography, 85(3) (July), 241-251. Recuperado de http://www.jstor.org/stable/40573705

Deaton, Angus (1997). The Analysis of Household Surveys. A Microeconometric Approach to Development Policy. Washington, D.C.: The World Bank. https://doi.org/10.1596/ 0-8018-5254-4

Doss, Cheryl (2013). Intrahousehold bargaining and Resource Allocation in Developing Countries. The World Bank Research Observer, 28(1), 52-78. https://doi.org/10.1093/ wbro/lkt001

Duffy, Mignon (2007). Doing the Dirty Work: Gender, Race, and Reproductive Labor in Historical Perspective. Gender and Society, 21(3), 313-336. https://doi. org/10.1177/0891243207300764

Fakih, Ali y Walid Marrouch (2012). Determinants of Domestic Worker's Employment; Evidence from Lebanese Household Survey Data. IZA Discussion Paper 6822.

Garavito, Cecilia (2015). Evolución del mercado de trabajo doméstico remunerado en el Perú. Documento de Trabajo 407. Departamento de Economía de la Pontificia Universidad Católica del Perú.

Garavito, Cecilia (2017). Oferta de trabajo del hogar remunerado en el Perú: 2007-2014. Cuadernos de Economía, 36(72), 265-299. https://doi.org/10.15446/cuad.econ. v36n72.57471

García, Luis (2006). Child Labor, Home Production and the Family Labor Supply. Revista de Análisis Económico, 21(1), 59-79. 
García, Luis (2007). Who does the Chores? Estimation of a Household Production Function in Peru. Lima: Departamento de Economía de la Pontificia Universidad Católica del Perú.

Gronau, Reuben (1977). Leisure, Home Production and Work - The Theory of the Allocation of Time Revisited. Journal of Political Economy, 86(6), 1099-1123. https://doi. org/10.1086/260629

Hersh, Joni y Leslie S. Stratton (1997). Housework, Fixed Effects, and Wages of Married Workers. The Journal of Human Resources, 32(2), Spring, 285-307. https://doi.org/10.2307/146216

Instituto Nacional de Estadística e Informática (2011). Encuesta Nacional de Uso del Tiempo 2010. Principales resultados. Lima: Ministerio de la Mujer y Desarrollo Social.

Klaveren, C. van, Maasen van den Brink, H. y B. van Praag (2011). Intra-Household Work Timing: The Effect on Joint Activities and the Demand for Child Care. IZA Discussion Paper 5636. https://doi.org/10.2139/ssrn.1809651

Kyriazidou, Ekaterini (1997). Estimation of a Panel Data Sample Selection Model. Econometrica, 65(6), 1335-1364. https://doi.org/10.2307/2171739

Lan, Pei-Chia (2003). Maid or Madam? Filipina Migrant Workers and the Continuity of Domestic Labor. Gender and Society, 17(2), April, 187-208. https://doi. org/10.1177/0891243202250730

Lundberg, Shelly y Robert A. Pollak (2007). The American Family and Family Economics. WP 12908, National Bureau of Economic Research. https://doi.org/10.3386/w12908

Mattingly, Doreen (2012). The Home and the World: Domestic Service and International Networks of Caring Labor. Annals of the Association of American Geographers, 91(2), June, 370-386. https://doi.org/10.1111/0004-5608.00249

Organización Internacional del Trabajo (2013). Trabajo doméstico remunerado en el Perú. Situación y perspectivas en función del Convenio 189 y la Recomendación 201 de la OIT. Documento de Trabajo. Lima: OIT. Recuperado el 17/11/14 de http://www.ilo.org/wcmsp5/groups/ public/---americas/---ro-lima/documents/publication/wcms_213173.pdf

Stancanelli, Elena G.F. y Leslie S. Stratton (2010). Her Time, His Time, or the Maid's Time: And Analysis of a Demand for Domestic Work. IZA Discussion Paper 5253, October.

Suen, Wing (1994). Market-Procured Housework: The Demand for Domestic Servants and Female Labor Supply. Labor Economics, 1(September), 289-302. https://doi. org/10.1016/0927-5371(94)90014-0

Documento recibido el 6 de agosto de 2018 y aprobado el 16 de noviembre de 2018 